\title{
Carnap's Logical Probability and Free Will Dilemma
}

\section{Paweł Pruski}

Institute of Philosophy, Jagiellonian University, Cracow, Poland

Email: minoo.music@gmail.com

How to cite this paper: Pruski, P. (2022). Carnap's Logical Probability and Free Will Dilemma. Open Journal of Philosophy, 12, 133-145.

https://doi.org/10.4236/ojpp.2022.121009

Received: January 29, 2022

Accepted: February 21, 2022

Published: February 24, 2022

Copyright $\odot 2022$ by author(s) and Scientific Research Publishing Inc. This work is licensed under the Creative Commons Attribution International License (CC BY 4.0).

http://creativecommons.org/licenses/by/4.0/

\begin{abstract}
Pondering the question of free will in the context of probability allows us to take a fresh look at a number of old problems. We are able to avoid deterministic entrapments and attempt to look at free will as an outcome of the entire decision-making system. In my paper, I will argue that free will should be considered in the context of a complex system of decisions, not individual cases. The proposed system will be probabilistic in character, so it will be embedded in the calculus of probability. To achieve the stated goal, I will refer to two areas of Carnap's interest: the relationship between free will and determinism, and the probability-based decision-making system. First, I will present Carnap's compatibilist position. On this basis, I will show how free will can be examined on deterministic grounds. Then I will present Carnap's probabilistic project-the so-called logical interpretation of probability. In addition to presenting its characteristics and functionality, I will argue for its usefulness in the context of decision analysis and its immunity to problems associated with determinism. Finally, I will show how the two mentioned elements can be combined, as a result of which I will present a concept for a probabilistic analysis of free will. In this context, I will identify free will with the individual characteristics of the system. My main aim is to present the theme of free will in the light of a formal analysis based on probability rather than metaphysical assumptions.
\end{abstract}

\section{Keywords}

Probability, Free Will, Determinism, Logical Interpretation of Probability

\section{Introduction}

What do probability and free will have in common? Colloquially speaking, "probability" is the expectation of a specific outcome of an event with an as yet undetermined result. We cannot unambiguously determine the result of a future event, 
so we determine the probability of its occurrence. This may be the result of insufficient data, a complicated system of dependencies, or the random nature of the event. In some cases, we simply do not have all the data about the system, e.g. a coin toss (information on the force of the throw, air resistance, etc.). In others, the event is probabilistic in nature within the realm of quantum processes (we are unable to determine the result but not because of a lack of data). On the other hand, free will presupposes that we have agency over our actions. The result of our actions is not a foregone conclusion-we decide the outcomes of specific events ourselves. If we have free will, we are able to make our own decisions. Otherwise, our actions would be a foregone conclusion-our future choices would be no more than consequences of deterministic cause-and-effect chains, over which we have no control. Our sense of agency would only be an illusion.

Both the concept of probability and free will assume that the world is open to a certain set of possibilities. Apart from determined causal sequences, there is room for chance, and our decisions are not completely determined by the current state of the system. They are free. When we look at the philosophical debate about free will, we see that various attempts have been made to defend it. Some prove that free will cannot be consistent with determinism (incompatibilists). Others try to show that there is no contradiction between free will and determinism (compatibilists). Carnap follows the second path (e.g., Carnap, 1966). The first part of this paper (I) is devoted to the characterization of Carnap's compatibilistic position. In this part, I will present the arguments for Carnap's views and alternative solutions for the defense of free will. As a consequence, a picture of the problems faced by both compatibilists and incompatibilists will emerge. We will see that implementing free will within the modern scientific outlook is an extremely difficult undertaking.

The reference to Carnap's probabilistic system (e.g., Carnap 1950), to which the second part of the text (II) is devoted, will be helpful in the context of explaining the function of probability on a semantic basis. It will show how we can create a multi-stage, probabilistic world description system as the basis for decision-making processes. In other words, I will present how our decisions can be a consequence of the application of the theory of probability. I will show that using probability, we can not only model decisions, but also introduce the so-called constant dispositions or rationality. All this information will be used in the third part of the text (III), where it will help in understanding the probabilistic analysis of free will.

In the last part of the text (III), I will present my arguments against considering decisions based on free will as single decision points. I will show how, in reference to Carnap's probabilistic proposal (II), we can consider the freedom of the will (I) in a broader context. We will learn how some of the problems related to free will analysis can be omitted if we treat it as a set of decisions based on probability. Contrary to many classical solutions, which mostly refer to metaphysics, I will show how to defend free will on the basis of probabilistic decision theory. 


\section{Free Will Dilemma}

The subject of free will is one of the central themes both in history and in modern philosophy (e.g., Dilman, 1999; Griffith, 2022; Grgić \& Pećnjak, 2018). After centuries of deliberations and despite the enormous development of science, the question of whether our will is indeed free still remains open. We are still searching for arguments which could allow us to reconcile the image of the world around us with the freedom of our will. On the one hand, we have the laws of physics that explain the processes that occur in our world. On the other hand, if we want to call our will free, we should have the ability to make choices without being limited by various factors. With the goal of this paper in mind, we will start our analysis in the first half of the 20th century. During this period, free will became much more difficult to reconcile with the evolving knowledge of the surrounding world. On the one hand, the idea collided with the deterministic worldview (classical mechanics). A question arose-why should our body, and the brain in particular, obey different laws than other particle objects? If, using classical mechanics, we can predict the movement of objects (planet positions, driving a car, falling objects), why should man be excluded from these laws? On the other hand, a space emerged to serve as a refuge for free will-the indeterministic quantum world. If certain processes occurring in the micro-world are not subject to deterministic laws, maybe it is in this area that we will find space for free will? The idea seems extremely tempting, but before we get into it, let's take a step back and go back to the topic of determinism. To start, let's consider the first problem-free will clashing with determinism, which we can define as follows.

Determinism: given the past at any time and the laws governing the universe, there is only one possible future. Whatever happens is therefore inevitable, it cannot but occur, given the past and laws (Kane \& Sartorio, 2021: p. 4).

One of the most explicit descriptions of the coexistence of determinism and free will is found in Laplace (see Laplace, 1951). The following quote shows how a deterministic description of the world translates into knowledge about its description.

We ought then to regard the present state of the universe as the effect of its anterior state and as the cause of the one which is to follow. Given for one instant an intelligence which could comprehend all the forces by which nature is animated and the respective situation of the beings who compose it-an intelligence sufficiently vast to submit these data to analysis-it would embrace in the same formula the movements of the greatest bodies of the universe and those of the lightest atom; for it, nothing would be uncertain and the future, as the past would be present to its eyes (Laplace, 1951: p. 4).

Thus, the state of each system at a later time is uniquely determined by its initial data set. In this context, the world around us resembles a domino-once it's set in motion, it moves in a specific direction. There is no room for chance or any spontaneous change that could violate this order. Particles follow a specific 
trajectory, and objects composed of them are subject to the laws of classical mechanics. Each subsequent state of the world is determined by an earlier one. So if we live in a world of physical objects and belong to this group ourselves, we are subject to the same deterministic laws. Our body, our brain in particular, is composed of the same atoms as the rest of the world. A fundamental question arises-should we regard our decisions in the same way, since they they are the result of our brain functions? Is free will also trapped in a deterministic chain of cause and effect? It is not difficult to guess that this is the point where an extensive debate begins. We are, however, interested in Carnap's way of thinking on this matter. In his opinion, determinism does not contradict the freedom of the will. In Laplace's vision, such freedom is achievable. This view is also one of the main ideas behind compatibilism.

Free choice is a decision made by someone capable of foreseeing the consequences of alternate action and choosing that which he prefers. There is no contradiction between free choice understood in this way and determinism, even of the strong classical type (Carnap, 1966: p. 333).

Moreover, Carnap believed that determinism is necessary for the implementation of free will. His justification for this argument refers to causal regularity. In this approach, determinism is a guarantee by which a link between our choice and its consequences can be established. Thanks to causality, we know that when we make a choice, we must expect certain consequences. When we choose between different courses of action and we know their consequences, it is a conscious choice. But if these actions were to have any random consequences, our choice would hardly be considered truly free. We could just as well replace it with a coin toss. Our freedom requires access to predictable alternatives. It requires a certain awareness of what will happen as a result of a particular decision. So let's examine another element. My choice is about predictable consequences, but is my decision truly free? Let's see what Carnap proposes.

Carnap begins defending his position with an example. In Philosophical Foundation of Physic (Carnap, 1966) he tells the story of a friend who likes some of Bach's compositions. By inviting him to a concert (where the above-mentioned compositions will be performed), we can be almost positive that he will accept the invitation and go to the performance.

Suppose that he actually comes with me, as I had expected. Was he compelled to go? No, he went of his own free will. He is never freer, in fact, than when given a choice of this sort (Carnap, 1966: p. 2019).

According to Carnap, the choice made by his friend is fully compatible with Laplace's view. Even if the knowledge of all the information about the universe made it possible to predict that he would go to the concert, we cannot say that he went there under duress. The opposite of freedom is not determinism, but an external limitation of action. Free will is defined as a state in which a person is free to act according to his or her own motivation, i.e., is not forced or restricted by outside factors. In other words, while a person may often be free to act on a motive, the nature of that motive is determined. So, free will and determinism 
are compatible-you can believe both without falling into logical inconsistency (e.g., Berofsky, 1987, Campbell, 1997, Vihvelin, 2013). Of course, many authors (e.g., Kane, 2005, Ginet, 1997) disagree with Carnap's and other compatibilists' theories. In their view, free will requires more than free action. The lack of a sense of compulsion does not mean that the decision is free. The core of the allegations formulated by incompatibilists can be reduced to the following argument. A person acts of her own free will only if she is its ultimate source. If determinism is true, no one is the ultimate source of her actions. Therefore, if determinism is true, no one acts of her own free will.

Perhaps more subtlety is required in our approach. Let us try to take advantage of the possibility of plunging into a quantum micro-world, as mentioned in the introduction. Perhaps in this indeterministic area we will find a space for free will. Carnap warns against such a course of action, and his suggestion is as follows:

The indeterminacy in quantum mechanics has no observable effect on what happens to a stone when each man throws it, because the stone is an enormous complex consisting of billion articles. In the micro world with which human beings are concerned, the indeterminacy of quantum mechanics plays no role (Carnap, 1966: p. 222).

In some sense, we can agree with the above statement. Human beings function in a macro-world which is subject to the laws of Newtonian mechanics. Perhaps we should put a thick red line between the micro- and macro-worlds, categorically separating these spaces.

But other philosophers have objected that quantum-level indeterminism does not end up amounting to macro-level indeterminism. In other words, just because a single atom may not behave deterministically, this does not mean that neurons, which are composed of many molecules (and thus even more atoms), will behave indeterministically. On the bigger level, maybe indeterminism does not amount to much and things are pretty well determined (Griffith, 2022: p. 116).

Let us assume, however, that we are very inquisitive and we want to abide by 20th century intuitions and look for a space for free will in the quantum micro-world. First things first, our decisions result from the activity of our brain, which consists of a neural system. This system is made up of a collection of specialized cells which remain in a complex structural relationship with each other. Finally, let's assume that our decision depends on how some indeterminate quantum state unfolds, as part of a neural system. If the micro-particle $\mathrm{M}$ takes the $\mathrm{x}$ position, we perform action $\mathrm{A}$, if it takes the y position-we perform action $\mathrm{B}$. It turned out that the micro-particle $\mathrm{M}$ took the $\mathrm{x}$ position, and we performed action A. Bear in mind that this process took place in an undetermined microworld. We were not able to clearly define the future position of the particle in an earlier time. Have we managed to defend free will? Not at all. The problem is as follows-we cannot point to intentionality at any stage. Our decision is a coincidence rather than a result of our will. Thus, if we extend Carnap's argument and admit the influence of the micro-world on the macro-world (in the area of 
decision-making) - the obtained result calls into question the fact that indeterminism is a proper area for the realization of free will.

Finally, at the third stage, we may corroborate this impression by reflecting on what Indeterminism (or the failure of Determinism) would bring to the table. We come to appreciate that Indeterminism wouldn't boost our free will, compared with Determinism, because all it would do is inject an element of randomness or pure chance that cannot enhance our control. That completes the motivation for Compatibilism (Kane \& Sartorio, 2021: p. 93).

Now that we have obtained an outline of Carnap's compatibilistic position, let us move on to the topic of probability. As we have seen, the combination of free will and determinism raises a number of doubts. Perhaps probability will allow us to create a space for free will to exist? Before we do that, however, let's explain once again why we will refer to it. We will see how the probabilistic model proposed by Carnap can become the basis of a decision-making system that avoids clashing with determinism. Let's remember that the Carnap proposal is a pioneering project. Currently, many branches of decision theory are based on the theory of probability. These ideas have many practical applications. Our interest lies in a slightly different approach. In the last part of the text (III) we will see how the above model works in the analysis of the concept of free will.

\section{Logical Probability and Decision}

In the introduction, we defined "probability" based on common intuitions. To better understand Carnap's position, we must first see how the above term is interpreted in terms of philosophy. In mathematics, probability is regarded as an undefined primary concept. The calculus of probability helps to calculate the chance of a certain event occurring. In philosophy, it occurs in association with many interpretations, and thus, many views on the nature of probability (e.g., Childers, 2013, Gillies, 2000, Skryms, 2000). What is the meaning of this concept? What is probability? What do probabilistic judgments relate to? The answers to the above questions depend on the choice of a specific interpretation of probability. This is a choice of great importance, as it will result in serious consequences. Carnap concentrated on the development of the so-called logical interpretation of probability.

The logical interpretation regards probability as an epistemic notion pertaining to our knowledge of facts rather than to facts themselves. Compared to the "classical" epistemic view of probability forged by Pierre Simon de Laplace, this approach stresses the logical aspect of probability, and regards the theory of probability as part of logic (Galavotti, 2011: p. 153).

This project was directly related to the attempt to create inductive logic (e.g., Carnap, 1950), and at a later stage tools used in decision theory (e.g., Carnap, 1971). In this context probability is determined on the semantic level, and specific sentences are the elements of relation. In other words, probability is a measure of logical relationship between sentences. Like Keynes (Keynes, 1921), Car- 
nap repeatedly emphasizes that probabilistic sentences do not say anything about the world, they only describe the relationships that occur within. Thus, semantics are imposed on a specific ontological model. This fact is especially important in the context of relationship to determinism, which we will deal with later in the text.

The model proposed by Carnap describes a simple universe. Thanks to this, we are able to observe how the semantic levels are created. In Logical Foundations of Probability Carnap constructs semantics based on a model with a finite $\left(L_{N}\right)$ and an infinite $\left(L_{\infty}\right)$ number of atomic sentences. Their structure is based on first-order logic and includes:

- logical conjunctions: $\neg, \wedge, \vee, \rightarrow, \leftrightarrow$

- quantifiers: $\forall, \exists$

- individual names: $a_{1}, a_{2} \ldots a_{n}$

- simple unary predicates: $P_{1}, P_{2} \ldots P_{n}$

- compound unary predicates: $Q_{1}, Q_{2} \ldots Q_{n}$

Despite simple foundations, the proposed semantics allows for the expression of virtually any description of the universe. As I will soon demonstrate, this enables us to create descriptions of alternative, possible worlds. So let's see how subsequent structures are created from the above-mentioned elements.

The so-called Q-predicates are built from predicates, i.e. conjunctions of all predicates (without repetitions). Each of the predicates included in the Q-predicate contains (or does not contain) negation, which is reflected in the fact that a given item has (or does not have) a specific feature. Each state name (representing an item) must be related to a specific predicate (representing a feature) that is within the scope of the Q-predicate (it cannot both have and not have a specific feature at the same time). The total of Q-predicates divides the whole universe into so-called Q-sets, which gather individuals belonging to the same Q-predicate. The total of Q-predicates divides the whole universe into so-called Q-sets, which gather individuals belonging to the same Q-predicate. Q-predicates and Q-sets are components of two key types of descriptions: state description and structure description. Each state description (S) consists of a series of state names and their assigned Q-predicates.

We will see that in relation to each system expressible in $L$-as opposed to more complex linguistic systems-every possible state can be expressed by a sentence or a class of sentences expressed in the form of the so-called individual description (Carnap, 1950: p. 70).

The result is a complete description of one of the possible worlds. Each state description defines one possible world (possessing an optimal description in the context of a given language model). We see that the scope of the formula tells us in which of the possible worlds our formula will be true. For example, a state description may take the following form:

$$
S i \Leftrightarrow Q 2_{1}\left(a_{1}\right) \wedge Q 2_{2}\left(a_{2}\right) \wedge Q 3_{3}\left(a_{3}\right) \wedge Q 1_{4}\left(a_{4}\right)
$$

with the help of state descriptions, we can designate another important parame- 
ter-the logical scope of a sentence. If sentence $\mathrm{p}$ follows from sentence $q$, then the logical range of $q$ is contained in the range of $p$. The opposite situation means that there is no relation between $p$ and $q$. All other values between $[0,1]$ mean that their logical ranges overlap to some extent. As a result, it is possible to establish a weight (measure) of each sentence (language $L_{N}$ or $L_{\infty}$ ) such that it is equal to the alternative of specific state descriptions. Thanks to the logical range of sentences, it is possible to determine the metric relation between sentences. In Logical Foundations of Probability Carnap proposes that the assurance measure should be closely related to logical probability. The aim is to probabilistically determine the degree of overlap between logical ranges of sentences. Within the discussed system, the relation is represented by the confirmation function. We are able to assign weight to individual elements and then create descriptions that allow us to estimate the probability.

A project such as this can be used, for example, to analyze decision-making processes. Carnap did just that in The Basic System of Inductive Logic (Carnap, 1971). To put it simply-each previous decision is based on the components that make up the subsequent metrically verifiable series.

The new point of view on the nature of logical probability can be called decisionistic, because within it the logic of induction begins to be considered not as a confirmation theory, but as the theoretical basis for rational decisions, with nonepistemic decisions involving the acceptance or rejection of hypotheses, but practical ones concerning certain material activities.

A key factor in the proposed characteristics is the so-called initial credence function, known as $C r_{0}$. It is a representation of the subject X's credence level at time $t_{0}$ (while it does not have any information yet). $C r_{0}$ is defined by four conditions.

(a) $E_{1} \ldots E_{n}$ are possible observational data

(b) $K_{n}$ is defined according to formula $K_{n}=\bigcap_{i=1}^{n} E_{i}$

(c) $C r_{0}$ fulfills all rationality requirements for a credence function

(d) Applying the $K_{n}$ values converts $C r_{0}$ to $C r_{n}$

The second important type of function is $C r_{0}^{\prime}$, known as credibility function, denoted by the Cred symbol. The Cred function is derived from the $C r$ function:

$$
C r_{n}(H)=C r_{0}^{\prime}\left(H / K_{n}\right)=C r_{0}^{\prime}(H \cap K) / C r_{0}(K)
$$

Carnap characterizes the credibility function as follows:

The trust function $H$ with respect to another judgment $A$, for person $X$ means the degree of belief that that person has with respect to $H$ if the total knowledge of the world was A (Carnap, 1968: p. 262).

The subject reconstructed by Carnap is subject to some idealizations. First, it has perfect memory. In order to determine $C r_{x}$ it is necessary to remember the data that occurred from the first sequence of $t$. Carnap proposes to assume $C r_{0}$ or Cred (credibility) as the basic factor for conditioning rationality. The above selection is conditioned by the reference to the so-called permanent dispositions, expressing inclinations that, in total, affect the image of the rationality of the 
subject. Carnap justifies the shift from a function of $C r$, which could serve as a prime determinant of rationality towards Cred, as follows:

For judging whether a person is intellectually rational or not, it is better to look at his credibility function than at his credence functions. While the credence functions merely reflect his momentary beliefs at various times, his credibility function expresses his underlying permanent disposition for forming and changing his beliefs under the influence of his observations (Carnap, 1968: p. 262).

To sum up. The above project shows how to create a probabilistic system that can be used in decision-making areas. Of course, Carnap's proposition is one of many that are based on the calculus of probability. Currently, various theoretical decision models are being developed, utilizing different interpretations of probability (e.g., Steele \& Stefánsson, 2015). Carnap's project is innovative in nature, and in addition it contains several elements that allow the introduction of additional parameters into the system. As we have seen, we can use it to model initial beliefs or permanent dispositions. In the background of the project there is also the possibility of determining rationality. This element is widely discussed in the context of the so-called Dutch Book Arguments (e.g., Vineberg, 2016). The reference to the axiomatics of probability is one of the methods of formal modeling of rationality. Thus, we get the possibility to determine individual decisions according to their rationality. We saw how to build a decision-making system based on the theory of probability. For any specific decision, the result is based on the calculation of probability resulting from the structure of the entire system (semantics). Bearing in mind the above elements, let's see what is the relationship of the described project to the topic of determinism and free will, as mentioned in the first part of this paper (I). Can we call decisions based on the calculus of probability free?

\section{Probabilistic Decision and Free Will}

In this part of the text let us adopt the following strategy. First, let's see whether the logical interpretation of probability described in the second part of this paper (II) and the decision theory based on it clash with determinism. Next, let's find out what are the benefits of applying a probabilistic decision-making system to the subject of free will. So let's start by confronting determinism. In other words, how do we reconcile the fact that events are necessarily determined by those that came before with the fact that we assign them a certain probability of occurrence? In the light of determinism, their fate is sealed, and in the light of probabilism, they have a certain chance of occurrence. In my opinion, the answer is yes-the above standpoints can coexist with each other. As we saw in the first part of the text, Carnap is an advocate of compatibilism, according to which it is possible to combine determinism with free will. Interestingly, although the author himself does not explicitly present this information anywhere, his probabilistic system (described in the second part of the text) also avoids colliding with determinism. The argument goes as follows. 
Logical probability is constructed on a semantic basis, so it is ontologically separated from empirical structures (subject to specific physical laws). From our point of view, the key fact is that the resulting judgments and subsequent descriptions operate "parallel" to empiricism. The logical interpretation of probability is used to create semantic descriptions of the world. Even if we agree with the logic-based analysis of determinism proposed by Schlick (Schlick, 1931), Carnap's proposal remains safe. According to this understanding of determinism, only one future is possible due to logic (a statement about a future event is either true or false). This form of determinism is based, among others, on bivalence (each sentence is true or false). The presented project, however, is based on a probabilistic assessment. Apart from extreme values (true, false) it allows all intermediate states described by probability. In this light, the future remains open. There is no clash between the probabilistic description and the ontology it refers to.

So let's change the perspective and focus on the next question that arises directly from the issue of determinism. Are we able to unequivocally predict the results in a system constructed this way? In my opinion, the answer is no. First of all, unique descriptions (individual/statistical) are created each and every time. Even if we assume that the model is based on a finite $L_{N}$ language, we still cannot unequivocally predict the results based on the input data. This situation resembles the one we can observe in modern chess programs, where despite the finite number of possibilities (resulting from the specificity of the game), we are not able to predict a specific move. Variable weights assigned to specific descriptions that generate the result are important. We should remember that additional elements also play an important role in Carnap's project. As we saw in (II), within the discussed project we can introduce e.g. initial credence functions or permanent dispositions. Consequently, each subsequent action within the system contributes to the creation of its unique character. Thanks to this, each system becomes more and more unique with time. Certain weights are determined, instructions are fixed and new information is adopted, modifying the system. The outlined process is consistent with the preference-building process that we deal with when making decisions.

Let us look at a few more elements that I believe make the probabilistic approach attractive in analyzing the subject of free will. First, let's look at two diagrams:

The first diagram $\left(d_{1}\right)$ shows the typical decision analysis we deal with in most considerations regarding decisive moments in the context of free will. We choose the point $t_{0}$ and we wonder if the choice of any of the alternatives will be volitional (Figure 1). Of course, previous preferences are taken into account, but the decisive moment is at time $t_{0}$. Even if we have additional information at the indicated point (see Carnap's example about Bach's listener), there is no clear correlation. We are unable to trace the history of a decision without falling into a determined causal sequence. The bivalent analysis of the previous decision nodes does not seem to reflect the actual decision-making process. 


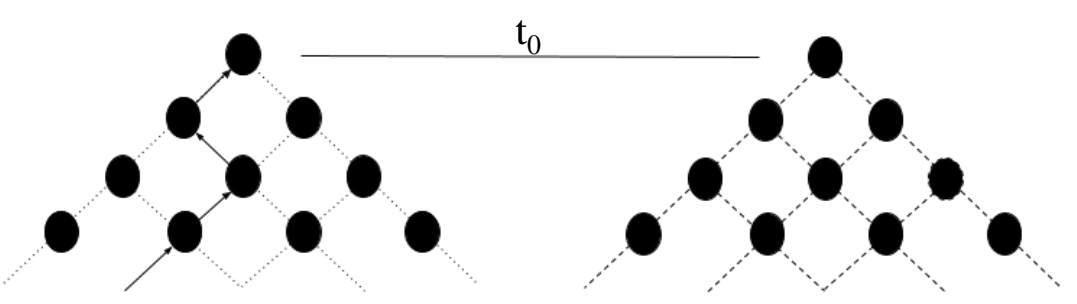

Figure 1. Two-valued decision tree $\left(d_{1}\right)$ and probabilistic decision tree $\left(d_{2}\right)$.

Now let's look at the same decision in a more complex structure $\left(d_{2}\right)$. Suppose the nodes preceding $t_{0}$ are assigned a certain probability weight (Figure 1). The system is probabilistic. We are dealing with a more subtle description of the story showing the weights assigned to individual points. We can see what degree of beliefs accompanied a given decision. Historical decisions did not stem from bivalency - they had different probabilities. So we are dealing with a full gray scale, not just a black and white image. Additionally, if new information is added to the system, it will be updated smoothly. As we have seen, Carnap's project has the ability to adopt new descriptions directly affecting state and statistical descriptions as well as credence functions and fixed dispositions. When making a decision, we move around the chosen area of the diagram (in accordance to the weight of probability nodes). We see very clearly how our decision is the result of our previous choices and dispositions. Why is it, that at point $t_{0}$ we are going to do this thing and not the other. Could we do otherwise? Yes. We are embedded in a fluid system. Do we feel any compulsion to behave a certain way? No. Of course, it may happen that as a result of external pressure our decision will not be free, but if such an event occurs it will be immediately visible in the system. The result (our decision) will be inconsistent with previous probabilities. If the result is historically inconsistent, there is a chance that our choice was due to external pressure or constraint. A fundamental question arises-would we be able to define a decision-making process categorized as a probabilistic system as free? Where exactly does free will fit in such a system?

\section{Conclusion}

The probabilistic analysis of free will proposed in this paper prompts a number of consequences. To begin with, let's answer the question about the nature of freedom in a system constructed in this way. In the proposed model, "freedom" is created holistically. It results from an individual, unique model that is formed over time. It is created on the basis of the information it contains, the initial function and permanent dispositions. The freedom of decision is determined by the overall shape of the system in question. Each subsequent deliberation or set of information influences its shape. Within different systems, we obtain different, unique decision outcomes. Why is he buying coffee at time $t_{0}$ ? Because the decision-making process took a certain path (through certain nodes) within its own system. Could he have done otherwise? Yes. It is possible that other parameters will be taken into account while making the decision, or that different 
weights will be assigned. We have at our disposal a finite amount of time, an individual system and preferences. We are not considering a single decision point, but also its history. The individual decision points (including the earlier ones) remain active. Contrary to the bivalent system-preferences can be altered in the analyzed proposal. The fact that a given decision is "mine" results from having an individual system. The same decision is considered free when it is at no stage determined by previous elements. The subject's decisions result from a dynamic system that guarantees free decisions. Thus, my proposal is in line with the compatibilist movement. Free will analyzed in probabilistic terms does not collide with determinism.

This proposal shifts the discussion over free will from the disciplines of metaphysics, ethics or theology to the realm of modern science. In the analysis of certain decisions, we do not refer to the metaphysical, but to the theory of probability and semantics. We get a metric tool that allows us to formally describe any given event. The presented model may be helpful in the analysis of the decisions of entities, but it can also be used in a wider application. For example, free will can be considered in relation to artificial intelligence. This thesis may be controversial, but in the light of its dynamic development, it is possible that we will at some point face this dilemma. Problems related to consciousness remain a separate issue, but the tool itself is perfectly practical. Of course, this project (based on Carnap's probabilistic ideas) presents an extremely simple model, but the ideas on display may form the basis for a more advanced proposal. It is altogether possible to add more elements or improve selected elements of the formal apparatus. Thus, probability may be one of the keys to solving the puzzle of free will.

\section{Acknowledgements}

This paper is the result of the research project No. 2013/09/N/HS1/00902 funded by the National Science Center.

\section{Conflicts of Interest}

The author declares no conflicts of interest regarding the publication of this paper.

\section{References}

Berofsky, B. (1987) Freedom from Necessity. Routledge \& Kegan Paul.

Campbell, J. (1997). A Compatibilist Theory of Alternative Possibilities. Philosophical Studies, 88, 319-330. https://doi.org/10.1023/A:1004280421383

Carnap, R. (1950). Logical Foundations of Probability. University of Chicago Press.

Carnap, R. (1966). Philosophical Foundations of Physics: An Introduction to the Philosophy of Science. Basic Books.

Carnap, R. (1968). Inductive Logic and Inductive Intuition. Studies in Logic and the Foundations of Mathematics, 51, 258-314. https://doi.org/10.1016/S0049-237X(08)71047-4

Carnap, R. (1971). A Basic System of Inductive Logic, Part I. In R. Carnap, \& R. C. Jeffrey 
(Eds.), Volume 1 (pp. 33-165). University of California Press. https://doi.org/10.1525/9780520334250-003

Childers, T. (2013). Philosophy and Probability. Oxford University Press.

Dilman, I. (1999). Free Will-An Historical and Philosophical Introduction. Routledge

Galavotti, M. (2011). The Modern Epistemic Interpretations of Probability: Logicism and Subjectivism. Handbook of the History of Logic, 10, 153-203. https://doi.org/10.1016/B978-0-444-52936-7.50005-7

Gillies, D. (2000). Philosophical Theories of Probability. Routledge.

Ginet, C. (1997). Freedom, Responsibility, and Agency. The Journal of Ethics, 1, 85-98. https://doi.org/10.1023/A:1009764120516

Grgić, F. \& Pećnjak D. (2018). Free Will \& Action. Springer Nature https://doi.org/10.1007/978-3-319-99295-2

Griffith, M. (2022). Free Will the Basics (2nd ed.). Routledge.

Kane, R. (Ed.) (2005). The Oxford Handbook of Free Will. Oxford University Press.

Kane, R. H., \& Sartorio C. (2021). Do We Have Free Will? Routledge.

Keynes, J. M. (1921). A Treatise on Probability. Macmillan.

Laplace, P. S. (1951). A Philosophical Essay on Probabilities (English Translation of the 6th French Edition). Dover.

Schlick, M. (1931) Die Kausalität in der gegenwärtigen Physik. Naturwissenschaften, 19, 145-162. https://doi.org/10.1007/BF01516406

Skryms, B. (2000). Choice and Chance (4th ed.). Wadsworth, Inc.

Steele, K., \& Stefánsson, H. O. (2015). Decision Theory. Stanford Encyclopedia of Philosophy.

Vihvelin, K. (2013). Causes, Laws, \& Free Will. Oxford University Press. https://doi.org/10.1093/acprof:oso/9780199795185.001.0001

Vineberg, S. (2016). Dutch Book Arguments. https://doi.org/10.1093/obo/9780195396577-0301

https://plato.stanford.edu/entries/dutch-book 